European Journal of Physics, Vol. 30, № 2, 259-270 (2009)

\title{
Linearization of conservative nonlinear oscillators
}

\author{
A. Beléndez ${ }^{(1)}$, M. L. Álvarez ${ }^{(1)}$ E. Fernández ${ }^{(2)}$ and I. Pascual ${ }^{(2)}$
}

(1) Departamento de Física, Ingeniería de Sistemas y Teoría de la Señal.

Universidad de Alicante. Apartado 99. E-03080 Alicante. SPAIN

(2) Departamento de Óptica, Farmacología y Anatomía.

Universidad de Alicante. Apartado 99. E-03080 Alicante. SPAIN

E-mail: a.belendez@ua.es

Corresponding author: A. Beléndez

Phone: +34-6-5903651

Fax: +34-6-5903464 


\begin{abstract}
A linearization method of the nonlinear differential equation for conservative nonlinear oscillators is analyzed and discussed. This scheme is based on the Chebyshev series expansion of the restoring force which allows us to obtain a frequency-amplitude relation which is valid not only for small but also for large amplitudes and, sometimes, for the complete range of oscillation amplitudes. Some conservative nonlinear oscillators are analyzed to illustrate the usefulness and effectiveness of the technique.
\end{abstract}

Keywords: Nonlinear oscillators; Approximate solutions; Chebyshev polynomials. 


\section{Introduction}

Nonlinear oscillator models have become increasingly important not only in many fields of physics and engineering, but also they are of significant importance in other areas [1]. Physical and mechanical oscillatory systems are often governed by non-linear differential equations. It is well known that sometimes a nonlinear equation of this type is linearized by retaining the first term of the Taylor series expansion of the restoring force $F(x)$ in the neighbourhood of the equilibrium point and dropping all but the leading term, obtaining $F(x) \approx-k x[2]$. This approximation is only valid for small amplitudes and corresponds to the familiar harmonic restoring force. This procedure yields acceptable results for many cases, but not only it is unable to show the amplitude dependence of the oscillation frequency but also not all restoring forces may be treated is this manner. As Mohazzabi [2] pointed out, contrary to the general claim that small oscillations in any nonlinear oscillator can be approximately treated in terms of simple harmonic motion, there are many oscillating systems for which this approximation is not valid. This means that to linearize these nonlinear oscillators using the Taylor series expansion is not possible. However, in this paper we can see that this does not imply that the nonlinear differential equations of conservative oscillators can't be approximated by a linear differential equation in which the approximate frequency depends on the oscillation amplitude. To do this approximation the Chebychev series expansion of the restoring force is used instead of the Taylor one [3, 4]. Some examples of conservative nonlinear oscillators are analyzed and we can see the approximate frequencies obtained are valid not only for small but also for large values of the oscillation amplitude and, in some situations, these approximate frequencies are valid for the complete range of oscillation amplitude, including the limiting cases of amplitude approaching zero and infinity. Excellent agreement of the approximate frequencies with the exact ones will be demonstrated and discussed.

Another important contribution of this paper is to clarify some important conceptual errors about nonlinear oscillators. Firstly, it is possible to have nonlinear oscillators whose behaviour is (a) linear for small values of the oscillation amplitude and nonlinear for large amplitudes, (b) nonlinear for the complete range of oscillation amplitude (known as intrinsically or truly nonlinear oscillators), (c) nonlinear for small amplitudes and linear for large values of the oscillation amplitudes, (d) linear for both for small as large oscillation amplitudes and nonlinear for intermediate values of the oscillation amplitude. And secondly, and contrary to the general claim, a conservative nonlinear oscillatory system 
can be always approximately treated in terms of simple harmonic motion, but with an approximate frequency that depends on the oscillation amplitude obtained by linearizing the nonlinear differential equation not using the Taylor expansion but the Chebyshev series expansion [4]. We think this is very important for students of Classical Mechanics courses at university level.

\section{Formulation and solution method}

Consider the simplest nonlinear conservative autonomous system encountered in the theory of oscillations with one degree of freedom, whose motion is governed by the following second order differential equation

$$
\frac{\mathrm{d}^{2} x}{\mathrm{~d} t^{2}}+f(x)=0
$$

with initial conditions

$$
x(0)=A, \quad \frac{\mathrm{d} x}{\mathrm{~d} t}(0)=0
$$

We suppose that the nonlinear function $f(x)$ is odd, i.e. $f(-x)=-f(-x)$ and satisfies $x f(x)>0$ for $x \in[-A, A], x \neq 0, A$ being the amplitude of motion. It satisfies that $x=0$ is the equilibrium position of the system and this oscillates symmetrically about its equilibrium point so that $x \in[-A, A]$. The motion is assumed to be periodic and the problem is to determine the angular frequency of oscillation and corresponding solution as a function of the system parameters and the amplitude $A$.

In order to derive simple analytical formulae we expand the function $f(x)$ in Taylor series around the equilibrium point $x=0$ and we obtain

$$
f(x)=\sum_{n=0}^{\infty} \frac{f^{(2 n+1)}(0)}{(2 n+1) !} x^{2 n+1}=\sum_{n=0}^{\infty} a_{2 n+1} x^{2 n+1}
$$

where 


$$
f^{(2 n+1)}(0)=\left(\frac{\mathrm{d}^{2 n+1} f(x)}{\mathrm{d} x^{2 n+1}}\right)_{x=0}
$$

and we have taken into account that the function $f(x)$ is odd and then the series expansion contains only terms involving odd powers of $x$. For small values of $x$ (and then of $A$ ) it is possible to do the following linear approximation

$$
f(x)=a_{1} x=\omega_{0}^{2} x
$$

where we have taken into account that $a_{1}$ is a positive constant in order to have an oscillatory motion and $\omega_{0}$ is the so-called linear angular frequency. It is well-known that $\omega_{0}$ does not depend on the oscillation amplitude $A$. Substituting Eq. (5) into Eq. (1) we obtain the wellknown liner differential equation for the simple harmonic oscillator

$$
\frac{\mathrm{d}^{2} x}{\mathrm{~d} t^{2}}+\omega_{0}^{2} x=0
$$

whose solution is

$$
x(t)=A \cos \omega_{0} t
$$

In many text books one can read that for small values of the oscillation amplitude, $A$, it is possible to replace the original nonlinear equation in (1) by the linear equation in (6) that approximates the original nonlinear equation giving useful results for small amplitudes. However this affirmation is not always correct [2]. The reason is that there are many oscillatory physical problems for which the elastic restoring forces, for small values of the displacement, are not dominated by a linear term and the linear approximation given in Eq. (6) is not valid even for small amplitudes. These systems are known as intrinsically nonlinear oscillators and Mohazzabi gave some examples of them in several areas including elasticity, electrodynamics, and gravitation [2]. We can conclude that the linear approximation given in Eqs. (5) and (6) —and commonly considered in some textbooks - is only valid for small oscillations of conservative nonlinear oscillators for which the restoring forces, for small 
values of the displacement, are dominated by a linear term. But, can we do another type of linearization for Eq. (1) which can be applied not only for small but also for large oscillation amplitudes as well as for intrinsically nonlinear oscillators? To answer this question we firstly introduce a reduced variable $y=x / A$ in Eqs. (1) and (2)

$$
\begin{aligned}
& \frac{\mathrm{d}^{2} y}{\mathrm{~d} t^{2}}+\frac{1}{A} f(A y)=0 \\
& y(0)=1, \quad \frac{\mathrm{d} y}{\mathrm{~d} t}(0)=0
\end{aligned}
$$

It is well-known that we can expand a function $f$ in terms of powers of $x$ (Taylor series) but also using other type of expansions. Denman [3] and Jonckheere [4] proposed the determination of the period of nonlinear oscillators by means the Chebyshev polynomials. Taking this into account, it is possible to expand the function $f(A y)$ in terms of Chebyshev polynomials of the first kind $T_{n}(x)$ as follows [5]

$$
f(A y)=\sum_{n=0}^{\infty} b_{2 n+1}(A) T_{2 n+1}(y)
$$

where the first polynomials are $[5,6]$

$$
T_{1}(y)=y, \quad T_{3}(y)=4 y^{3}-3 y, \quad T_{5}(y)=16 y^{5}-20 y^{3}+5 y, \quad T_{6}(y)=32 y^{6}-48 y^{4}+18 y^{2}-1
$$

and $[5,6]$

$$
b_{2 n+1}(A)=\frac{2}{\pi} \int_{-1}^{+1}(1-y)^{-1 / 2} f(A y) T_{2 n+1}(y) \mathrm{d} y
$$

which are amplitude dependent. In Eq. (10) we have taken into account that $f(x)$ is an odd function of $x$. A series expansion in terms of Chebyshev polynomials converges much faster than the Taylor expansion [4]. We can approximate Eq. (10) retaining only the first term as follows 


$$
f(A y)=b_{1}(A) T_{1}(y)=b_{1}(A) y=\frac{b_{1}(A)}{A} x
$$

where

$$
b_{1}(A)=\frac{2}{\pi} \int_{-1}^{+1}(1-y)^{-1 / 2} f(A y) T_{1}(y) \mathrm{d} y=\frac{2}{\pi} \int_{-1}^{+1}(1-y)^{-1 / 2} f(A y) y \mathrm{~d} y
$$

The nonlinear differential equation in (1) can be then approximated by the following linear differential equation

$$
\frac{\mathrm{d}^{2} x}{\mathrm{~d} t^{2}}+\omega^{2}(A) x=0
$$

where $\omega$ is the approximate angular frequency of the nonlinear oscillator and it can be obtained as follows

$$
\omega(A)=\sqrt{\frac{b_{1}(A)}{A}}
$$

and the corresponding approximate periodic solution is

$$
x(t)=A \cos [\omega(A) t]
$$

\section{Examples}

In this section, we present some examples to illustrate the usefulness and effectiveness of the linearization technique.

\section{1.- The Duffing oscillator}

This oscillator is governed by the following nonlinear differential equation

$$
\frac{\mathrm{d}^{2} x}{\mathrm{~d} t^{2}}+\omega_{0}^{2}\left(x+\varepsilon x^{3}\right)=0
$$


with initial conditions given in Eq. (2). Using the Taylor series expasion in Eq. (5) we obtain the following angular frequency

$$
\omega_{T}=\omega_{0}
$$

whereas if we use the Chebyshev series expansion in Eq. (16), the angular frequency is given as follows

$$
\omega_{C h}(A)=\omega_{0} \sqrt{1+\frac{3}{4} \varepsilon A^{2}}
$$

In Figure 1 we compare the nonlinear function $f(x) / \omega_{0}^{2}=x+\varepsilon x^{3}$ with its linearized approximations obtained using the Taylor series expansion, $f_{T}(x) / \omega_{0}^{2}=x$, and the Chebyshev series expansion, $f_{C h}(x) / \omega_{0}^{2}=\left(1+\frac{3}{4} A^{2}\right) x$.

On the other hand, direct integration yields the exact frequency $\omega_{e}(A)$ as $[1,7]$

$$
\omega_{e}(A)=\frac{\pi \omega_{0} \sqrt{1+\varepsilon A^{2}}}{2 K(m)}, \quad m=\frac{\varepsilon A^{2}}{2\left(1+\varepsilon A^{2}\right)}
$$

where $K(m)$ is the complete elliptic integral of the first kind. We can easily verify that

$$
\lim _{\varepsilon A^{2} \rightarrow \infty} \frac{\omega_{C h}(A)}{\omega_{e}(A)}=\frac{\sqrt{3}}{\pi} K(1 / 2) \approx 1.022205
$$

which is independent on the value of $\omega_{0}$. This means that the relative error of the approximate frequency tends to $2.2 \%$ when the oscillation amplitude tends to infinity. We can conclude that the approximation given in Eq. (20) is accurate not only for small but also for large oscillation amplitudes. 


\section{2.- The simple pendulum}

This is perhaps one of the systems more times analyzed approximately [8-15]. The nonlinear differential equation for the free, undamped simple pendulum is

$$
\frac{\mathrm{d}^{2} x}{\mathrm{~d} t^{2}}+\omega_{0}^{2} \sin x=0
$$

with initial conditions given in Eq. (2). Using the Taylor series expansion in Eq. (5) we obtain the following angular frequency $\omega_{T}=\omega_{0}=\sqrt{g / l}$, whereas if we use the Chebyshev series expansion in Eq. (16) the angular frequency is given as follows

$$
\omega_{C h}(A)=\omega_{0} \sqrt{\frac{2 J_{1}(A)}{A}}
$$

where $J_{1}(A)$ is the first-order Bessel function of the first kind. In Figure 2 we compare the nonlinear function $f(x) / \omega_{0}^{2}=\sin x$ with its linearized approximations obtained using the Taylor series expansion, $f_{T}(x) / \omega_{0}^{2}=x$, and the Chebyshev series expansion $f_{C h}(x) / \omega_{0}^{2}=\left(2 J_{1}(A) / A\right) x$.

Direct integration of Eq. (23) yields the exact frequency $\omega_{e}(A)$ as follows $[1,12]$

$$
\omega_{e}(A)=\frac{\pi \omega_{0}}{2 K(m)}, \quad m=\sin ^{2} \frac{A}{2}
$$

It is easy to verify that the relative errors of the approximate frequencies $\omega_{T}(A)$ and $\omega_{C h}(A)$ are less than $1 \%$ for $A<23^{\circ}$ and $A<117^{\circ}$, respectively.

\section{3.- Antisymmetric, constant force oscillator}

Suppose that a particle of mass $m$ moving in one dimension, $x$, has a state of stable equilibrium produced by a force that is directed toward the origin and has a constant magnitude $F(x)=-F_{0} \operatorname{sgn}(x)$ where $F_{0}$ is a positive constant and 


$$
\operatorname{sgn}(x)=\left\{\begin{array}{cc}
1, & x>0 \\
0, & x=0 \\
-1, & x<0
\end{array}\right.
$$

The nonlinear differential equation for this case is

$$
\frac{\mathrm{d}^{2} x}{\mathrm{~d} t^{2}}+\alpha^{2} \operatorname{sgn}(x)=0, \quad \alpha=\sqrt{\frac{F_{0}}{m}}
$$

with initial conditions given in Eq. (2).

One example of this nonlinear system consists of an infinite sheet of electric charge, with a uniform charge density and a small hole in the middle [2]. A particle with a electric charge $q$, opposite to the sheet, oscillates through the hole. Another example is the motion of a particle of mass $m$ rolling in a "V" shape trough in a constant gravitational field [1]. The arms of the "V" make equal angles with the horizontal plane and the origin of the (horizontal) $x$ coordinate is taken to be the point of interaction of the two arms.

The oscillations corresponding to Eq. (26) are intrinsically nonlinear and the function $\alpha^{2} \operatorname{sgn}(x)$ cannot be expanded in Taylor series. This implies that it is impossible to do the approximation given in Eq. (5). However, we can use the Chebyshev series expansion in Eq. (16) and the angular frequency is given as follows

$$
\omega_{C h}(A)=\frac{2 \alpha}{\sqrt{\pi A}}
$$

In Figure 3 we compare the nonlinear function $f(x) / \alpha^{2}=\operatorname{sgn}(x)$ with its linearized approximation obtained using the Chebyshev series expansion, $f_{C h}(x) / \alpha^{2}=\frac{4}{\pi A} x$.

Direct integration of Eq. (26) yields the exact frequency $\omega_{e}(A)$ as follows $[1,16-18]$ 


$$
\omega_{e}(A)=\frac{\pi \alpha}{2 \sqrt{2 A}}
$$

We can easily verify that

$$
\frac{\omega_{C h}(A)}{\omega_{e}(A)}=\frac{4 \sqrt{2}}{\pi^{3 / 2}} \approx 1.01590
$$

which is independent on the values of $A$ and $\alpha$. This means that the relative error of the approximate frequency is less than $1.6 \%$ for all oscillation amplitudes.

\section{4.- Mass attached to two stretched elastic springs}

The nonlinear differential equation for this case is $[1,19]$

$$
\frac{\mathrm{d}^{2} z}{\mathrm{~d} t^{2}}+\frac{2 k}{m} z-\frac{2 k l_{0} z}{m \sqrt{d^{2}+z^{2}}}=0
$$

with initial conditions given in Eq. (2). In Eq. (31) $m$ is the mass of the particle, $k$ is the spring constant and $l_{0}$ is the length of the each elastic wire when any force is applied. We assume that the movement of the particle is one-dimensional and this is constrained to move only in the horizontal $x$ direction (Figure 4). One of the ends of each wire is fixed a distance $2 d$ a part, and $d \geq l_{0}$. If $d=l_{0}$ the wires are not stretched for $x=0$ and there is no tension in each part of it. However, if $d>l_{0}$ the wire is stretched for $x=0$ and the tension in each part of the wire is $k\left(d-l_{0}\right)$. Defining $z=x d$ and $\lambda=l_{0} / d$, Eq. (31) can be re-written as follows $[20,21]$

$$
\frac{\mathrm{d}^{2} x}{\mathrm{~d} t^{2}}+\omega_{0}^{2}\left(1-\frac{\lambda}{\sqrt{1+x^{2}}}\right) x=0
$$

where $\omega_{0}=\sqrt{2 k / m}$. As $0<l_{0} \leq d$ it follows that $0<\lambda \leq 1$.

For large $x$, and for $0<\lambda \leq 1$, Eq. (32) approximates that of a linear harmonic oscillator 


$$
\frac{\mathrm{d}^{2} x}{\mathrm{~d} t^{2}}+\omega_{0}^{2} x=0 \quad \text { for } x>>1 \text { and } 0<\lambda \leq 1
$$

so, for large $A$, we have $\omega \approx \omega_{0}$. For small $x$, and for $0<\lambda<1$, the equation of motion also approximates that of a linear oscillator

$$
\frac{\mathrm{d}^{2} x}{\mathrm{~d} t^{2}}+(1-\lambda) \omega_{0}^{2} x=0 \quad \text { for } x<<1 \text { and } 0<\lambda<1
$$

and $\omega \approx \omega_{0} \sqrt{1-\lambda}$ for small $A$. This implies that the behaviour of this oscillator is linear for both for small as large oscillation amplitudes and nonlinear for intermediate values of the oscillation amplitude.

However, for small $x$, and for $\lambda=1$, Eq. (32) approximates that of an intrinsically nonlinear oscillator

$$
\frac{\mathrm{d}^{2} x}{\mathrm{~d} t^{2}}+\frac{1}{2} \omega_{0}^{2} x^{3}=0 \quad \text { for } x<<1 \text { and } \lambda=1
$$

and $\omega$ tends to zero when $A$ decreases $[2,20]$. Consequently, the angular frequency $\omega$ increases from $\omega_{0} \sqrt{1-\lambda}$ to $\omega_{0}$ as the initial value of $x(0)=A$ increases.

We can see that for $0<\lambda<1$ the behaviour of the nonlinear oscillator is linear both small as large oscillation amplitudes. However, for $\lambda=1$, this is an example of an intrinsically nonlinear oscillator for small amplitudes for which the linear approximation based on the Taylor expansion cannot be done for small values of the oscillation amplitude. However, for large values the system oscillates with simple harmonic motion.

We can consider the Chebyshev series expansion in Eq. (16) and the approximate angular frequency can be obtained follows

$$
\omega_{C h}(A)=\omega_{0}\left[1-\frac{4 \lambda}{\pi A^{2}}\left(\sqrt{1+A^{2}} E\left(\frac{A^{2}}{1+A^{2}}\right)-\frac{1}{\sqrt{1+A^{2}}} K\left(\frac{A^{2}}{1+A^{2}}\right)\right)\right]^{1 / 2}
$$


where $K(m)$ and $E(m)$ are the complete elliptic integrals of the first and the second kind, respectively, which are defined as follows

$$
\begin{gathered}
K(m)=\int_{0}^{\pi / 2} \frac{\mathrm{d} \theta}{\sqrt{1-m \sin ^{2} \theta}} \\
E(m)=\int_{0}^{\pi / 2} \sqrt{1-m \sin ^{2} \theta} \mathrm{d} \theta
\end{gathered}
$$

In Figure 5 we compare the nonlinear function $f(x) / \omega_{0}^{2}=\left(1-\frac{1}{\sqrt{1+x^{2}}}\right) x($ for $\lambda=1)$ with its linearized approximation obtained using the Chebyshev series expansion, $f_{C h}(x) / \omega_{0}^{2}=\left[\omega_{C h}^{2}(A) / \omega_{0}^{2}\right] x$.

Integrating Eq. (32) and using the initial conditions in Eq. (2), we arrive at

$$
\frac{1}{2}\left(\frac{\mathrm{d} x}{\mathrm{~d} t}\right)^{2}+\frac{1}{2} x^{2}-\lambda \sqrt{1+x^{2}}=\frac{1}{2} A^{2}-\lambda \sqrt{1+A^{2}}
$$

The exact frequency can then be derived as follows

$$
\omega_{e}(A)=\frac{\pi}{2} \omega_{0}\left[\int_{0}^{1} \frac{A \mathrm{~d} u}{\sqrt{A^{2}\left(1-u^{2}\right)-2 \lambda\left(\sqrt{1+A^{2}}-\sqrt{1+A^{2} u^{2}}\right)}}\right]^{-1}
$$

which must be solved numerically for each value of $A$.

As an example we only consider $\lambda=1$. In this situation, and for small values of the amplitude $A$, it is possible to take into account the following approximation [20]

$$
\omega_{e}(A) \approx \frac{\pi}{2} \omega_{0}\left[\int_{0}^{1} \frac{2 \mathrm{~d} u}{\sqrt{\left(1-u^{2}\right)\left(1+u^{2}\right)}}\left(\frac{1}{A}+\ldots\right)\right]^{-1} \approx \frac{\pi \sqrt{2} \omega_{0}}{4 K(1 / 2)} A+\ldots=0.59907 \omega_{0} A+\ldots
$$


For small values of $A$ it is also possible to do a power-series expansion of the approximate angular frequency $\omega_{C h}(A)($ Eq. (36)) and we obtain [20]

$$
\omega_{C h}(A) \approx \omega_{0} \sqrt{\frac{3}{8}} A+\ldots=0.61237 \omega_{0} A+\ldots
$$

This power-series expansion is done using MATHEMATICA. We can easily verify that

$$
\lim _{A \rightarrow \infty} \frac{\omega_{C h}(A)}{\omega_{e}(A)}=1
$$

and

$$
\lim _{A \rightarrow 0} \frac{\omega_{C h}(A)}{\omega_{e}(A)}=\frac{\sqrt{3}}{\pi} K(1 / 2) \approx 1.022205
$$

which means that the relative error of the angular frequency tends to $2.2 \%$ when $A$ tends to zero. We can conclude that $\omega_{C h}(A)$ in Eq. (36) gives excellent approximate frequencies for small as well large values of oscillation amplitude $A$.

\section{5.- The dynamically shifted oscillator}

Suppose that a particle of mass $m$ moving in one dimension, $x$, has a state of stable equilibrium produced by the nonlinear force $F(x)=-k x-k x_{0} \operatorname{sgn}(x)[2,22]$, where $k$ is a positive constant. The nonlinear differential equation for this case is

$$
\frac{\mathrm{d}^{2} x}{\mathrm{~d} t^{2}}+\omega_{0}^{2}\left[x+x_{0} \operatorname{sgn}(x)\right]=0, \quad \omega_{0}=\sqrt{\frac{k}{m}}
$$

with initial conditions given in Eq. (2). Parameter $x_{0}$ is the dynamic shift and it can be positive or negative, but in the last situation $A>2\left|x_{0}\right|$ [18].

Using the Chebyshev series expansion in Eq. (16) the angular frequency is given as follows 


$$
\omega_{C h}(A)=\omega_{0} \sqrt{1+\frac{4 x_{0}}{\pi A}}
$$

In Figure 6 we compare the nonlinear function $f(x) / \omega_{0}^{2}=x+x_{0} \operatorname{sgn}(x)$ with its linearized approximation obtained using the Chebyshev series expansion, $f_{C h}(x) / \omega_{0}^{2}=1+\frac{4 x_{0}}{\pi A} x$.

Direct integration of Eq. (26) yields the exact frequency $\omega_{e}(A)$ as follows $[2,22]$

$$
\omega_{e}(A)=\omega_{0}\left[1-\frac{2}{\pi} \sin ^{-1}\left(\frac{x_{0}}{A+x_{0}}\right)\right]^{-1}
$$

For small values of the amplitude $A$, it is possible to take into account the following approximation

$$
\omega_{e}(A) \approx \frac{\pi \omega_{0}}{2 \sqrt{2}} \sqrt{\frac{x_{0}}{A}}+\ldots=1.11072 \omega_{0} \sqrt{\frac{x_{0}}{A}}+\ldots
$$

For small values of $A$ it is also possible to do a power-series expansion of the approximate angular frequency $\omega_{C h}(A)($ Eq. (46)) and we obtain

$$
\omega_{e}(A) \approx \frac{2 \omega_{0}}{\sqrt{\pi}} \sqrt{\frac{x_{0}}{A}}+\ldots=1.12838 \omega_{0} \sqrt{\frac{x_{0}}{A}}+\ldots
$$

This power-series expansion is done using MATHEMATICA. We can easily verify that

$$
\lim _{A \rightarrow \infty} \frac{\omega_{C h}(A)}{\omega_{e}(A)}=1
$$

and

$$
\lim _{A \rightarrow 0} \frac{\omega_{C h}(A)}{\omega_{e}(A)}=\frac{4 \sqrt{2}}{\pi^{3 / 2}} \approx 1.01590
$$


which means that the relative error of the angular frequency tends to $1.6 \%$ when $A$ tends to zero. We can conclude that $\omega_{C h}(A)$ in Eq. (46) gives excellent approximate frequencies for the complete range of oscillation amplitude.

\section{Conclusions}

A linearization method for conservative nonlinear oscillators based on the Chebyshev series expansion of the restoring force [3] has been analyzed and a frequency-amplitude relationship has been obtained. Some examples of nonlinear oscillators have been approximately solved using this scheme and good results have been obtained, not only for small but also for large values of the oscillation amplitude. In some cases the approximate frequency gives accurate results for the complete range of amplitudes. Some major conclusions are: (a) the linearization of the nonlinear differential equation taking into account the Taylor series expansion of the restoring force can't be always done (some nonlinear oscillators are intrinsically nonlinear) and when this expansion is possible the linearization obtained is only valid for small amplitudes (the frequency does not depend on the oscillation amplitude), and (b) the linearization of the nonlinear differential equation is always possible if the Chebyshev series expansion of the restoring force is done, and (c) this scheme provides excellent approximations to the solution of these nonlinear systems with high accuracy not only for small but also for large values of the oscillation amplitude. Finally, in this paper not only did we reveal some interesting aspects of the study of nonlinear oscillators -such as the examples considered- or procedures for determining approximations to the periodic solutions of non-linear differential equations, but we also presented an application of one of these procedures to easily obtain analytical approximations for the frequency which have some pedagogical value to both graduate as well as undergraduate students. 


\section{References}

[1] Mickens R E 1996 Oscillations in Planar Dynamics Systems (Singapore: World Scientific).

[2] Mohazzabi P 2004 Theory and examples of intrinsically nonlinear oscillators $A m$. J. Phys. 72 492-8

[3] Denman H H 1969 An approximate equivalent linearization technique for nonlinear oscillations J. Appl. Mech. 36 358-60

[4] Jonckheere 1971 Determination of the period of nonlinear oscillations by means of Chebyshev polynomials ZAMM-Zeitschrift fur Angewandte Mathematik und Mechanik 55 389-93

[5] Abramowitz M. and Stegun I A (Eds.) 1972 "Orthogonal Polynomials", Ch. 22 in Handbook of Mathematical functions with Formulas, Grapas and Mathematical Tables, 9th printing. New York: Dover, pp. 771-802.

[6] Weisstein E W Chebyshev Polynomial of the First Kind. From MathWorld-A Wolfram web resource. http://mathworld.wolfram.com/ChebyshevPolynomialoftheFirstKind.html

[7] Amore P, Raya A and Fernández F M 2005 Alternative perturbation approaches in classical mechanics Eur. J. Phys. 26, 1057-63

[8] Parwani R R 2004 An approximate expression for the large angle period of a simple pendulum Eur. J. Phys. 25, 37-49

[9] Beléndez A, Hernández A, Márquez A, Beléndez T and Neipp C 2006 Analytical approximations for the period of a simple pendulum Eur. J. Phys. 27 539-51

[10] He J H 2006 Some asymptotic methods for strongly nonlinear equations Int. J. Modern Phys. B 10 1141-99

[11] Lima F M S and Arun P 2006 An accurate formula for the period of a simple pendulum oscillating beyond the small angle regime Am. J. Phys. 74 892-5 
[12] Beléndez A, Hernández A, Beléndez T, Neipp C and Márquez A 2007 Application of the homotopy perturbation method to the nonlinear pendulum Eur. J. Phys. 28 93-104

[13] Amore P, Valdovinos M C, Orneles G and Barajas S Z 2007 The nonlinear pendulum: formulae for the large amplitude period Rev. Mex. Fis. E 53 106-111

[14] Beléndez A, Hernández A, Beléndez T, Márquez A and Neipp C 2007 An improved 'heuristic' approximation for the period of a nonlinear pendulum: linear analysis of a nonlinear problem Int. J. Non-linear Sci. Numer. Simulat. 8 329-34

[15] Lima F M S 2008 Simple 'log formulae' for pendulum motion valid for any amplitude Eur. J. Phys. 29 1091-8

[16] Gatland I R 1991 Theory of an nonharmonic oscillator Am. J. Phys. 59 155-8

[17] Özis T and Yildirim A 2007 A comparative study of He's homotopy perturbation method for determining frequency-amplitude relation of a nonlinear oscillator with discontinuities Int. J. Nonlinear Sci. Numer. Simul. 8 243-8

[18] Beléndez A, Hernández A, Beléndez T, Neipp C and Márquez A 2008 Higher accuracy analytical approximations to a nonlinear oscillator with discontinuity by He's homotopy perturbation method Phys. Lett. A 372 2010-6

[19] Marion J B and Thornton S T 1995 Classical Dynamics of Particles and Systems (New York: Saunders)

[20] Beléndez A, Hernández A, Beléndez T, Álvarez M L, Gallego S, Ortuño M and Neipp, C 2007 Application of the harmonic balance method to a nonlinear oscillator typified by a mass attached to a stretched wire J. Sound Vib. 302 1018-29

[21] Shou D H 2008 Variational approach to the nonlinear oscillator of a mass attached to a stretched wire Phys. Scr. 77, art. 045006

[22] Hartmann W M 1986 The dynamically shifted oscillator Am. J. Phys. 54 28-31 


\section{FIGURE CAPTIONS}

Figure 1.- Comparison between the nonlinear function $f(x) / \omega_{0}^{2}$ with their linearized approximations obtained using the Taylor series expansion, $f_{T}(x) / \omega_{0}^{2}$, and the Chebyshev series expansion, $f_{C h}(x) / \omega_{0}^{2}$, for the Duffing oscillator with $\varepsilon=1$ and $A=1$.

Figure 2.- Comparison between the nonlinear function $f(x) / \omega_{0}^{2}$ with their linearized approximations obtained using the Taylor series expansion, $f_{T}(x) / \omega_{0}^{2}$, and the Chebyshev series expansion, $f_{C h}(x) / \omega_{0}^{2}$, for the simple pendulum with $A=$ $120^{\circ}$.

Figure 3.- Comparison between the nonlinear function $f(x) / \omega_{0}^{2}$ with its linearized approximation obtained using the Chebyshev series expansion, $f_{C h}(x) / \omega_{0}^{2}$, for the antisymmetric, constant force oscillator with $A=1$.

Figure 4.- A mass attached to two identical springs oscillating in the $x$-axis direction.

Figure 5.- Comparison between the nonlinear function $f(x) / \omega_{0}^{2}$ with its linearized approximation obtained using the Chebyshev series expansion, $f_{C h}(x) / \omega_{0}^{2}$, for a mass attached to two stretched elastic springs with $\lambda=1$ and $A=1$.

Figure 6.- Comparison between the nonlinear function $f(x) / \omega_{0}^{2}$ with its linearized approximation obtained using the Chebyshev series expansion, $f_{C h}(x) / \omega_{0}^{2}$, for the dynamically shifted oscillator with $x_{0}=0.4$ and $A=1$. 
FIGURE 1

- Exact -.-.-Chebyshev ...... Taylor

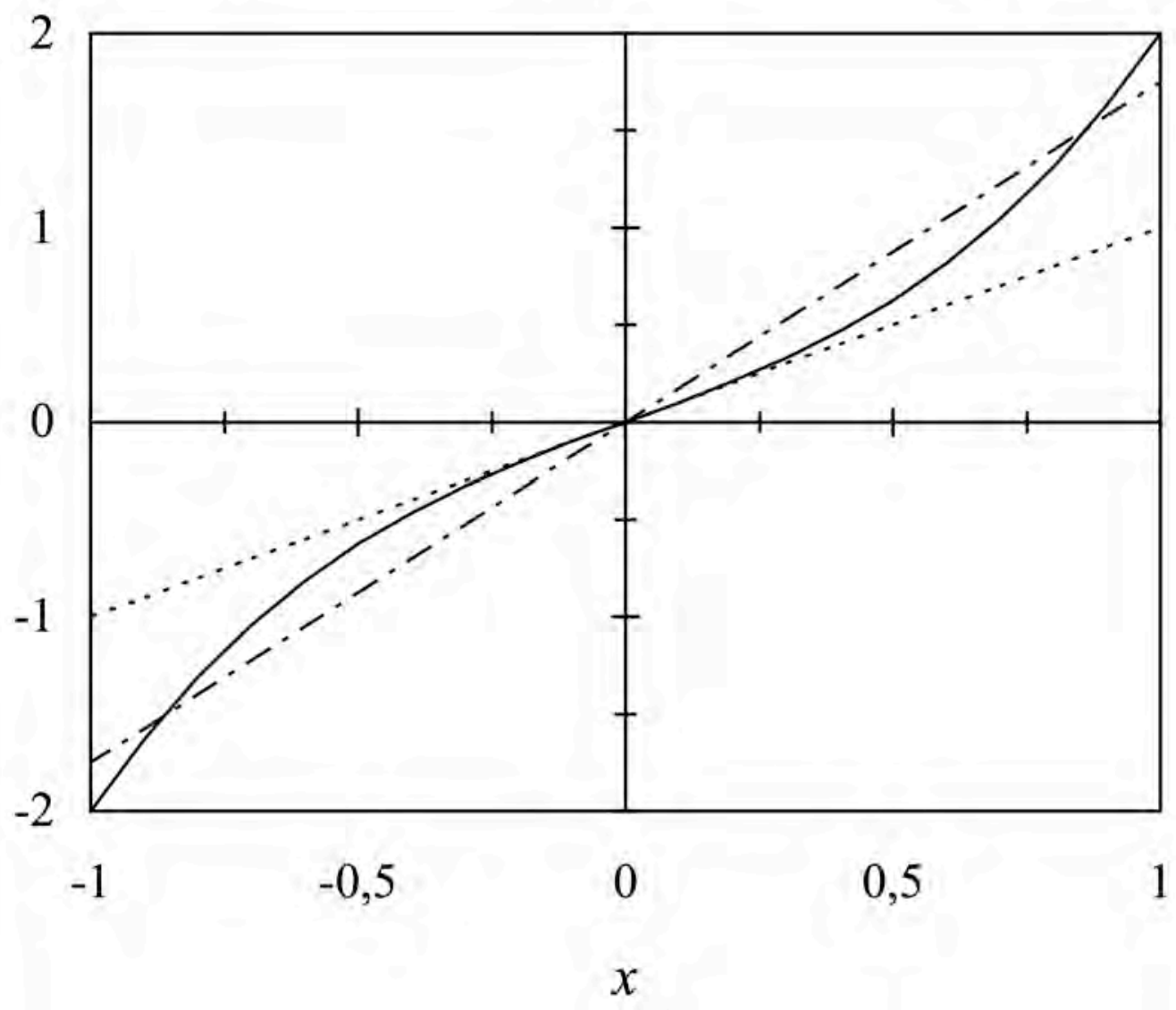


FIGURE 2

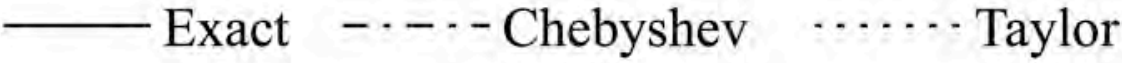

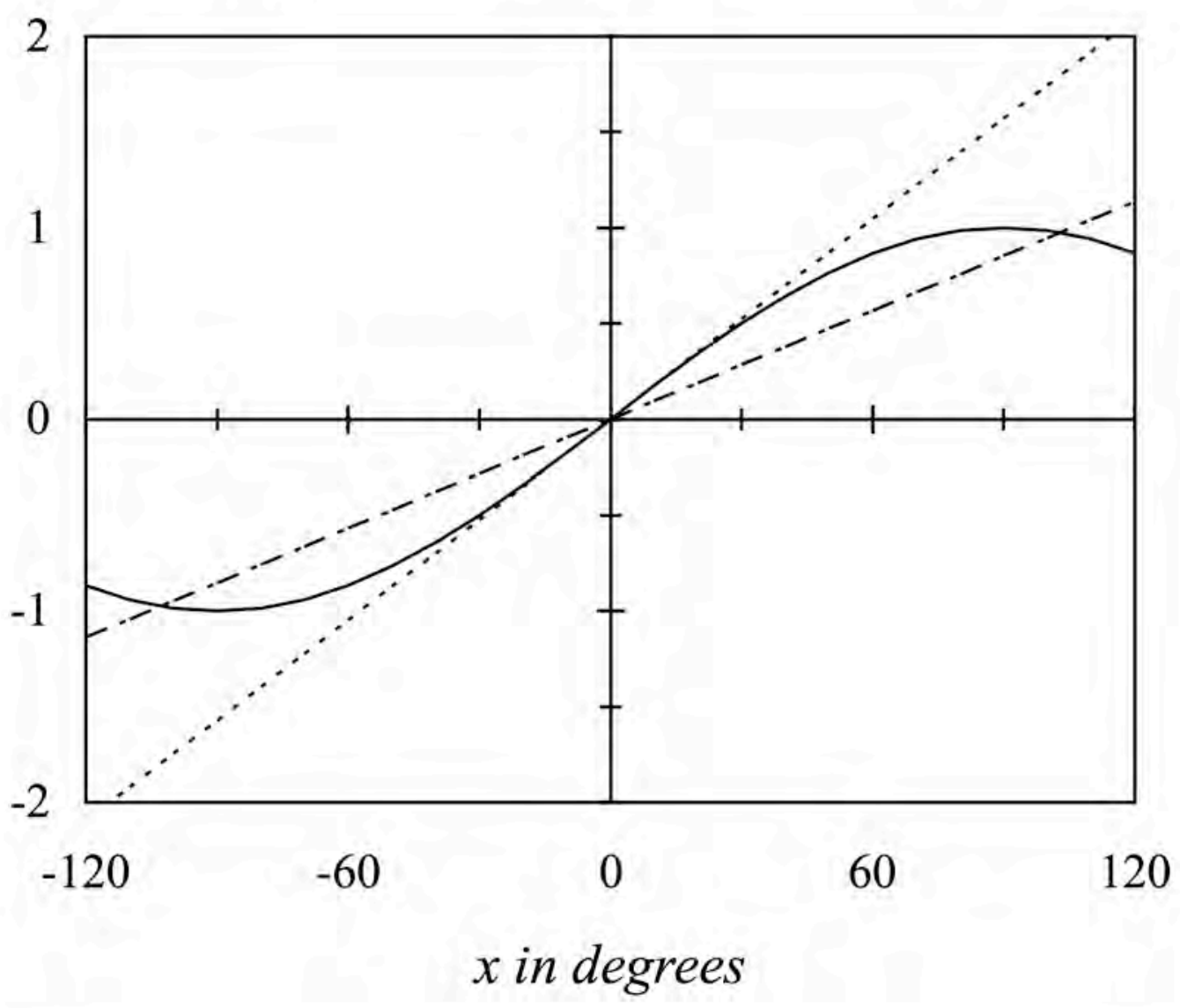


FIGURE 3

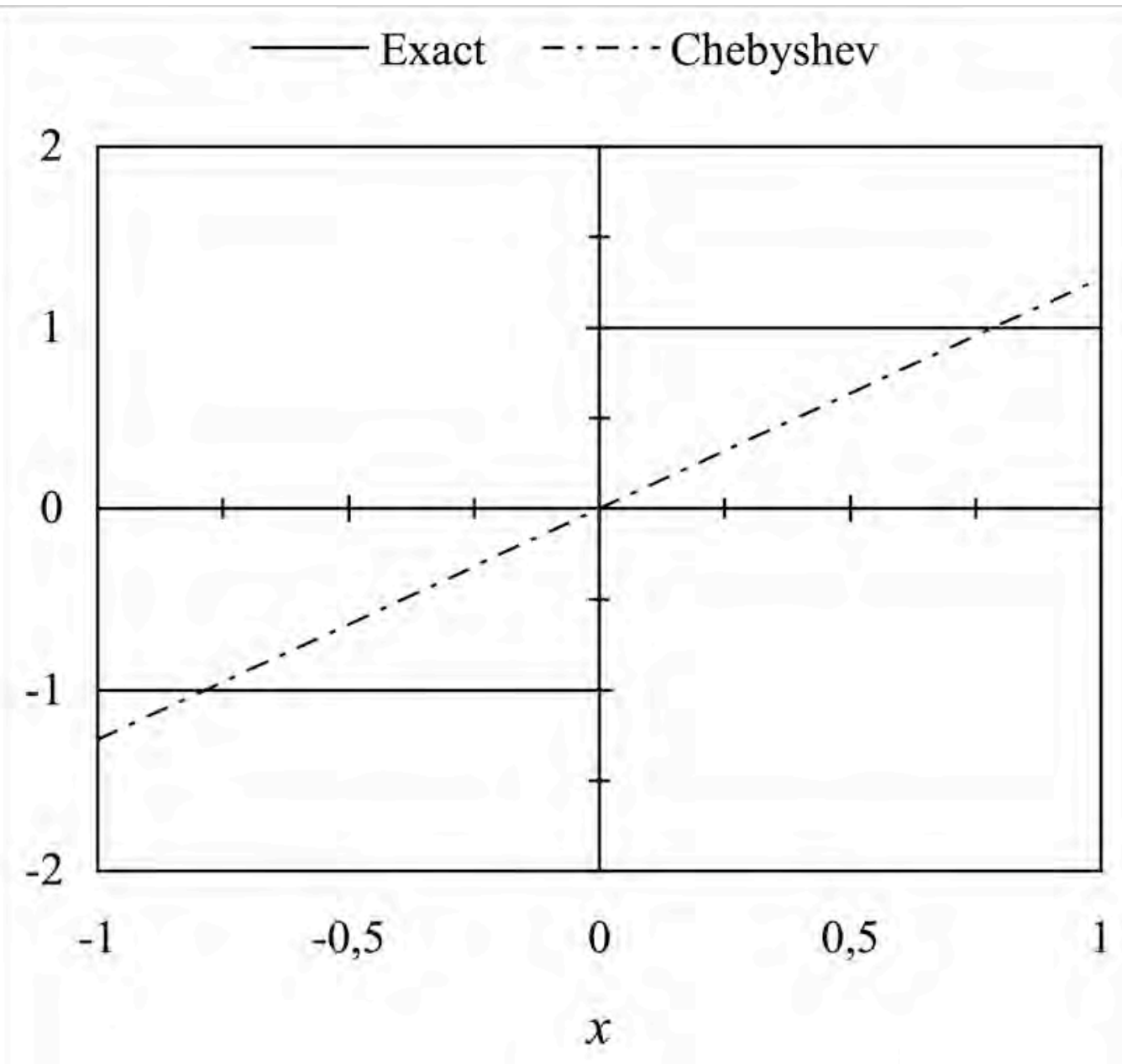


FIGURE 4
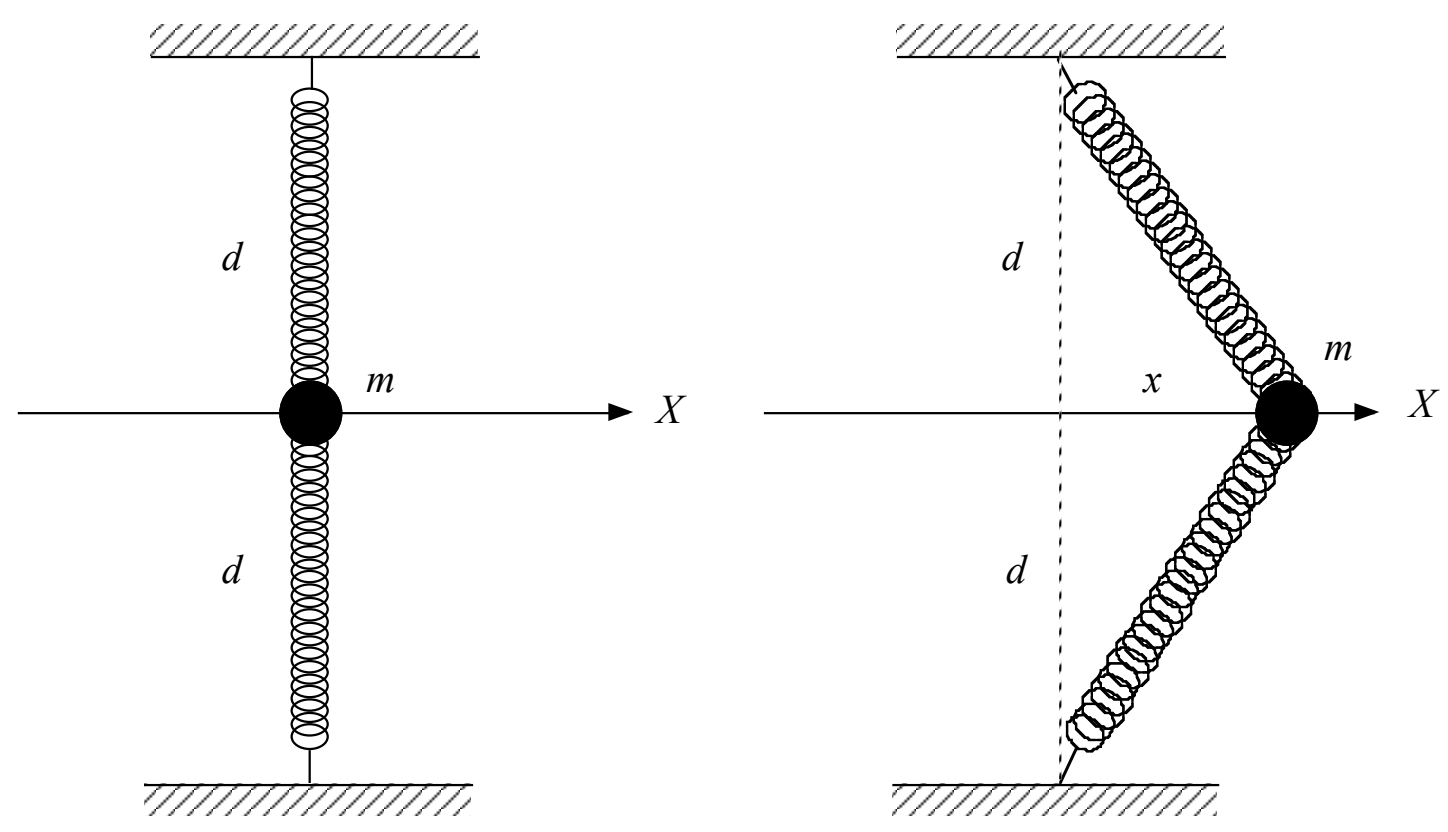
FIGURE 5

- Exact -.-. Chebyshev

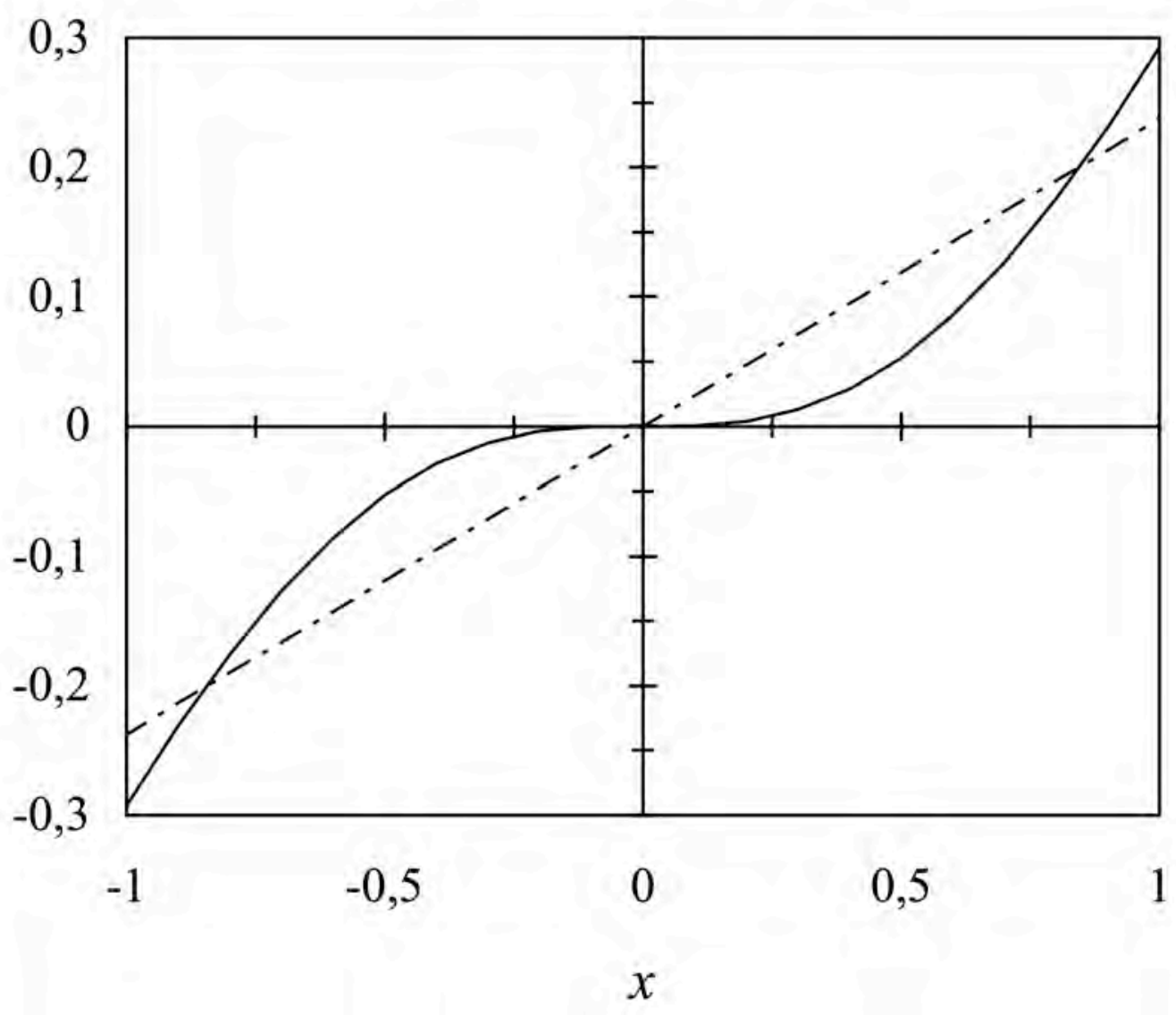


FIGURE 6

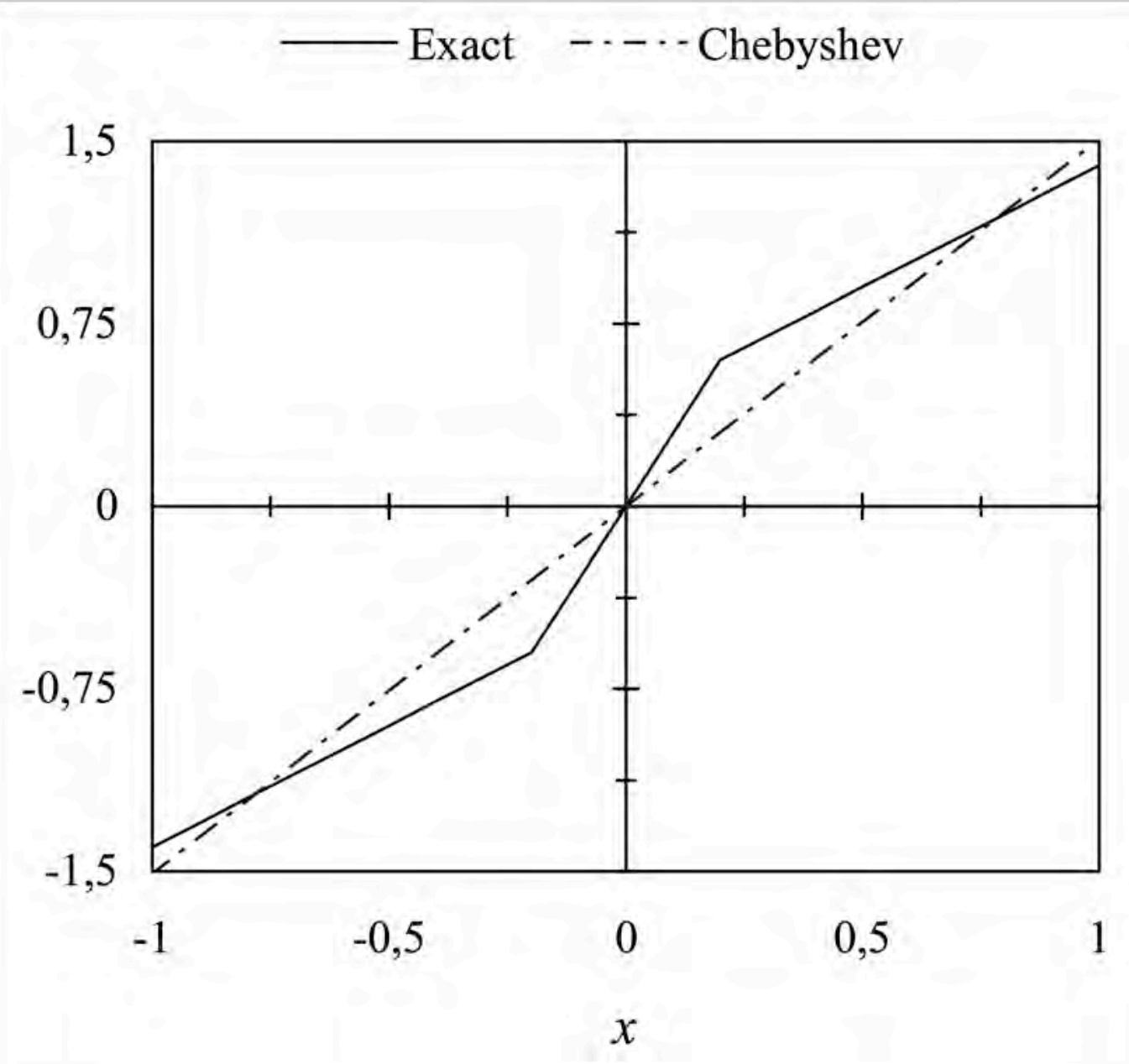

